\title{
SEMI-PARAMETRIC COVARIATE-MODULATED LOCAL FALSE DISCOVERY RATE FOR GENOME-WIDE ASSOCIATION STUDIES
}

By Rong W. ZabLOCKI ${ }^{\dagger, \ddagger}, R_{\text {ICHARD A. LEVINE }}^{\dagger}$ ANDREW J. SchorK $^{\S}$ Shujing XU ${ }^{\S}$ Yunpeng Wang ${ }^{\Uparrow}$ Chun C. FAN ${ }^{\S}$ And Wesley

K. ThOMPSON $\$, \|, * *, *$

San Diego State University ${ }^{\dagger}$, Claremont Graduate University ${ }^{\ddagger}$, University of California at San Diego ${ }^{\S}$, University of Oslo, Norway ", Institute of

Biological Psychiatryl, and The Lundbeck Foundation Initiative for Integrative Psychiatric Research**

\section{Supplement A: Conditional Posteriors and Gibbs Sampling} Algorithm.

1.1. Conditional posterior densities. The full conditional distributions for the unknown parameters may be obtained as follows. Throughout, $f(\cdot \mid \ldots)$ denotes the kernel probability density of a parameter conditioned on all other parameters and the data. First,

$$
\begin{aligned}
f\left(\boldsymbol{\alpha}_{\boldsymbol{m} \cdot \mid \ldots)} \mid \ldots \exp \left\{-\frac{1}{2 \tau_{m}^{2}}\left(\boldsymbol{\alpha}_{\boldsymbol{m}(\mathbf{2 : K})} \boldsymbol{\Omega}^{*} \boldsymbol{\alpha}_{\boldsymbol{m}(\mathbf{2 : K})^{T}}+\sum_{j=0 ; j \neq m}^{M}\left[\boldsymbol{\alpha}_{\boldsymbol{j}(\mathbf{2 : K})} \boldsymbol{\Omega}^{*} \boldsymbol{\alpha}_{\boldsymbol{j}(\mathbf{2 : K})}^{T}\right]\right)\right\}\right. \\
\prod_{i ; \delta_{i}=1} \prod_{k=1}^{K}\left[\left\{\frac{\exp \left(x_{i m} \alpha_{m k}+\boldsymbol{x}_{\boldsymbol{i ( - \boldsymbol { m } )}}^{\boldsymbol{T}} \boldsymbol{\alpha}_{(-\boldsymbol{m}) \boldsymbol{k}}\right)}{\sum_{l=1}^{K} \exp \left(x_{i m} \alpha_{m l}+\boldsymbol{x}_{\boldsymbol{i ( - \boldsymbol { m } )}}^{\boldsymbol{T}} \boldsymbol{\alpha}_{(-\boldsymbol{m}) \boldsymbol{l}}\right)}\right\}^{I\left(\eta_{i}=k\right)}\right] .
\end{aligned}
$$

The last part of (1.1) is equivalent to $\prod_{i ; \delta_{i}=1} \prod_{k=1}^{K}\left[\left\{\frac{\exp \left(\boldsymbol{x}_{\boldsymbol{i}}^{T} \boldsymbol{\alpha} \cdot \boldsymbol{k}\right)}{\sum_{l=1}^{K} \exp \left(\boldsymbol{x}_{\boldsymbol{i}}^{\boldsymbol{T}} \boldsymbol{\alpha} \cdot \mathbf{l}\right)}\right\}^{I\left(\eta_{i}=k\right)}\right]$, except that (1.1) separates the terms of the $m^{\text {th }}$ covariate from all the other covariates (denoted by a $-m$ subscript).

${ }^{*}$ To whom correspondence should be addressed. 
Second,

$$
\begin{aligned}
& f\left(\tau_{m}^{2} \mid \ldots\right) \propto\left(\tau_{m}^{2}\right)^{-\frac{K-1}{2}} \exp \left\{-\frac{1}{2 \tau_{m}^{2}} \boldsymbol{\alpha}_{\boldsymbol{m}(\mathbf{2 : K})} \boldsymbol{\Omega}^{*} \boldsymbol{\alpha}_{\left.\boldsymbol{m}(\mathbf{2 : K})^{T}\right\}}\right\}\left(\tau_{m}^{2}\right)^{\left(-\frac{\nu}{2}-1\right)} \exp \left(-\frac{\frac{\nu}{a_{m}}}{\tau_{m}^{2}}\right) \\
& \sim \text { Inverse Gamma }\left(\frac{K+\nu-1}{2}, \frac{\boldsymbol{\alpha}_{\boldsymbol{m}(\mathbf{2 : K})^{*} \boldsymbol{\alpha}_{\boldsymbol{m}(\mathbf{2 : K})^{T}}}}{2}+\frac{\nu}{a_{m}}\right) .
\end{aligned}
$$

Third,

$$
\begin{aligned}
& f\left(a_{m} \mid \ldots\right) \propto a_{m}^{-\frac{\nu}{2}} \exp \left(-\frac{\frac{\nu}{a_{m}}}{\tau_{m}^{2}}\right) a_{m}^{-\frac{1}{2}-1} \exp \left(-\frac{\frac{1}{A^{2}}}{a_{m}}\right) \\
& \sim \text { Inverse Gamma }\left(\frac{\nu+1}{2}, \frac{\nu}{\tau_{m}^{2}}+\frac{1}{A^{2}}\right) .
\end{aligned}
$$

Fourth,

$$
\begin{aligned}
& f\left(\sigma_{0}^{2} \mid \ldots\right) \propto\left(\sigma_{0}^{2}\right)^{-\left(\frac{N_{0}}{2}+a_{0}\right)-1} \exp \left\{-\frac{1}{\sigma_{0}^{2}}\left(\frac{\boldsymbol{z}_{\mathbf{0}}^{T} \boldsymbol{z}_{\mathbf{0}}}{2}+b_{0}\right)\right\} \\
& \sim \text { Inverse Gamma }\left(\frac{N_{0}}{2}+a_{0}, \frac{\boldsymbol{z}_{\mathbf{0}}^{T} \boldsymbol{z}_{\mathbf{0}}}{2}+b_{0}\right) .
\end{aligned}
$$

Fifth,

$$
f(\boldsymbol{\gamma} \mid \ldots) \propto \prod_{i=1}^{N}\left[\left\{\frac{\exp \left(\boldsymbol{x}_{\boldsymbol{i}}^{T} \boldsymbol{\gamma}\right)}{1+\exp \left(\boldsymbol{x}_{\boldsymbol{i}}^{T} \boldsymbol{\gamma}\right)}\right\}^{\delta_{i}}\left\{\frac{1}{1+\exp \left(\boldsymbol{x}_{\boldsymbol{i}}^{T} \boldsymbol{\gamma}\right)}\right\}^{1-\delta_{i}}\right] \exp \left(-\frac{\boldsymbol{\gamma} \Sigma_{\gamma}^{-1} \boldsymbol{\gamma}}{2}\right)
$$

The distributions in (1.1) and (1.5) do not take any standard distributional form.

1.2. Sampling Scheme. In GWAS, each SNP is coded as a count of the number of reference alleles (i.e., 0, 1, or 2). Since choice of reference allele is essentially random with respect to the outcome, the resulting distribution of $z$-scores is modeled as symmetric around zero. It is straightforward 
to allow for asymmetry if necessary in other applications of cmfdr. In the present case, in order to simplify the implementation, we fold both the null and non-null distributions at zero. Hence, the null distribution becomes a folded normal distribution with location 0 and scale $\sigma_{0}^{2}$, and the non-null distribution is constructed on the absolute value of $z$-scores. The parameters $\boldsymbol{\alpha}, \boldsymbol{\tau}^{2}, \boldsymbol{a}, \boldsymbol{\gamma}$ and $\sigma_{0}^{2}$ are sampled in turn from their full conditional distributions via a Gibbs sampler. At each iteration, $\sigma_{0}^{2}$ can be sampled directly from its posterior inverse gamma distribution; $\tau_{m}^{2}$ and $a_{m}$ can be sampled from their respective posterior inverse gamma distributions recursively from $m=0$ to M. The matrix parameter $\boldsymbol{\alpha}$ is generated through compoments $\boldsymbol{\alpha}_{\boldsymbol{m}}$. At each iteration, $\boldsymbol{\alpha}_{\boldsymbol{m}}$. may be updated recursively based on (1.1) for $m=0,1,2, \ldots, M$. While updating $\boldsymbol{\alpha}_{\boldsymbol{m}}$., all the other $\boldsymbol{\alpha}_{-\boldsymbol{m}}$. are treated as constant. This is the reason that formula (1.1) separates terms with and without $m$. Since both $\boldsymbol{\alpha}_{\boldsymbol{m}}$. and $\boldsymbol{\gamma}$ do not take standard form, variates are generated using multiple-try Metropolis-Hastings (MTMH) samplers (Givens and Hoeting, 2005). Multivariate- $t$ candidate distributions are used in the MTMH sampler with parameters obtained by maximizing $\boldsymbol{\alpha}_{\boldsymbol{m}}$. and $\gamma$ over (1.1) and (1.5) respectively. That is, the maximizers and the corresponding negative inverse Hessian of (1.1) and (1.5) are used as parameters in the MTMH routine.

Two indicators, $\boldsymbol{\delta}$ and $\boldsymbol{\eta}$, also require updating during each Gibbs iteration. At the global level for each test, we update $\delta_{i}$ to be either 1 or 0 based on the probabilities

$$
\begin{array}{r}
\frac{\exp \left(\boldsymbol{x}_{\boldsymbol{i}}^{T} \boldsymbol{\gamma}\right) f_{1}\left(\left|z_{i}\right| \mid \boldsymbol{\alpha}, \boldsymbol{x}_{\boldsymbol{i}}\right)}{\exp \left(\boldsymbol{x}_{\boldsymbol{i}}^{T} \boldsymbol{\gamma}\right) f_{1}\left(\left|z_{i}\right| \mid \boldsymbol{\alpha}, \boldsymbol{x}_{\boldsymbol{i}}\right)+f_{0}\left(\left|z_{i}\right| \mid \sigma_{0}^{2}\right)}, \text { and } \\
\frac{f_{0}\left(\left|z_{i}\right| \mid \sigma_{0}^{2}\right)}{\exp \left(\boldsymbol{x}_{\boldsymbol{i}}^{T} \boldsymbol{\gamma}\right) f_{1}\left(\mid z_{i} \| \boldsymbol{\alpha}, \boldsymbol{x}_{\boldsymbol{i}}\right)+f_{0}\left(\mid z_{i} \| \sigma_{0}^{2}\right)}
\end{array}
$$

The indicator $\boldsymbol{\delta}$ may be initialized by taking an upper percentile (e.g., upper $5 \%$ ) of the $\left|z_{i}\right|$ scores as 1 and 0 otherwise. At the local level, for each non-null 
test, we update $\eta_{i}$ to be one of the $(1,2, \ldots, K)$ based on the $K$ probabilities

$$
\begin{aligned}
& \frac{\exp \left(\boldsymbol{x}_{i, \delta_{i}=1}^{T} \boldsymbol{\alpha} \cdot \mathbf{1}\right) g_{1}\left(\left|z_{i, \delta_{i}=1}\right|\right)}{\sum_{k=1}^{K}\left[\exp \left(\boldsymbol{x}_{i, \delta_{i}=1}^{T} \boldsymbol{\alpha}_{\cdot \boldsymbol{k}}\right) g_{k}\left(\left|z_{i, \delta_{i}=1}\right|\right)\right]}, \\
& \frac{\exp \left(\boldsymbol{x}_{i, \delta_{i}=1}^{T} \boldsymbol{\alpha} \cdot \mathbf{2}\right) g_{2}\left(\left|z_{i, \delta_{i}=1}\right|\right)}{\sum_{k=1}^{K}\left[\exp \left(\boldsymbol{x}_{i, \delta_{i}=1}^{T} \boldsymbol{\alpha} \cdot \boldsymbol{k}\right) g_{k}\left(\left|z_{i, \delta_{i}=1}\right|\right)\right]}, \\
& \vdots \\
& \frac{\exp \left(\boldsymbol{x}_{i, \delta_{i}=1}^{T} \boldsymbol{\alpha} \cdot \boldsymbol{K}\right) g_{K}\left(\left|z_{i, \delta_{i}=1}\right|\right)}{\sum_{k=1}^{K}\left[\exp \left(\boldsymbol{x}_{i, \delta_{i}=1}^{T} \boldsymbol{\alpha}_{\cdot \boldsymbol{k}}\right) g_{k}\left(\left|z_{i, \delta_{i}=1}\right|\right)\right]} .
\end{aligned}
$$

If we have $L$ draws $\left\{\left(\boldsymbol{\alpha}^{(l)}, \boldsymbol{\tau}^{2(l)}, \boldsymbol{a}^{(l)}, \boldsymbol{\gamma}^{(l)}, \sigma_{0}^{2(l)}\right): 1 \leq l \leq L\right\}$ from the Gibbs sampler, then for each draw $l$,

$$
\operatorname{cmfdr}^{(l)}\left(z_{i}\right)=\frac{\pi_{0}\left(\boldsymbol{x}_{i} \mid \boldsymbol{\gamma}^{(l)}\right) f_{0}\left(\left|z_{i}\right| \mid \sigma_{0}^{2(l)}\right)}{\pi_{0}\left(\boldsymbol{x}_{i} \mid \boldsymbol{\gamma}^{(l)}\right) f_{0}\left(\left|z_{i}\right| \mid \sigma_{0}^{2(l)}\right)+\pi_{1}\left(\boldsymbol{x}_{i} \mid \boldsymbol{\gamma}^{(l)}\right) f_{1}\left(\mid z_{i} \| \boldsymbol{\alpha}^{(l)}, \boldsymbol{x}_{i}\right)} .
$$

Hence, an a posteriori estimate of $\operatorname{cmfdr}\left(z_{i}\right)$ for each test can be obtained. Due to the symmetry of $f_{1}$ and $f_{0}$, if two tests have the same covariates and same absolute value (opposite sign) of $z$-score, their cmfdr values will be the same. On the other hand, if two tests have identical $z$-scores but different covariates, their cmfdr values will be different. The algorithm has been implemented in the $\mathrm{R}$ statistical package ( $\mathrm{R}$ Core Team, 2016). The simulations were run on a cluster with 27 Dell PowerEdge C1100 nodes. Each node contains 75 gigabytes (GB) of RAM and dual Intel Xeon X5650 $2.66 \mathrm{GHz}$ processors with 6 cores for a total of $2025 \mathrm{~GB}$ of RAM and 324 cores. A MCMC chain of 18,000 iterations with 50,000 cases took about 20 hours for the semi-parametric cmfdr and about 12 hours for fdr. As for the real data application, all models were run on a Linux Intel Xeon E5$26602.20 \mathrm{GHz}$ processor with 20 cores for a total of $400 \mathrm{~GB}$ of memory. An example of computational resource allocation for a MCMC chain of 23,000 iterations with 74,800 SNPs is shown in Table 1 . There is no surprise that the semi-parametric model with covariates is more computationally expensive. 
TABLE 1

Computational resource allocation on three different models.

\begin{tabular}{lccc}
\hline & semi-parametric cmfdr & gamma cmfdr & fdr \\
\hline Virtual memory (megabyte) & 507 & 459 & 495 \\
Physical memory (megabyte) & 261 & 218 & 249 \\
Time to finish (hours) & 55 & 16 & 16 \\
\hline
\end{tabular}

\section{Supplement B: KEGG homo sapiens pathways with ALIGA- TOR $p$-values from three models (full list).}

Table 2: KEGG homo sapiens pathways with ALIGATOR p-values from three models (full list).

\begin{tabular}{|c|c|c|c|}
\hline Pathway & $\begin{array}{l}p \text {-values } \\
\text { (semi- } \\
\text { parametric) }\end{array}$ & $\begin{array}{l}p \text {-values } \\
\text { (gamma) }\end{array}$ & $\begin{array}{l}p \text {-values } \\
\text { (fdr) }\end{array}$ \\
\hline Axon guidance & $6.00 \mathrm{E}-04$ & 0.002 & 0.2046 \\
\hline Herpes simplex infection & $8.00 \mathrm{E}-04$ & 0.0268 & 1 \\
\hline Osteoclast differentiation & 0.0062 & 0.0192 & 1 \\
\hline Pentose phosphate pathway & 0.0096 & 0.5206 & 1 \\
\hline Tuberculosis & 0.01 & 0.0068 & 0.132 \\
\hline Leishmaniasis & 0.0162 & 0.0946 & 1 \\
\hline Antigen processing and presentation & 0.022 & 0.096 & 1 \\
\hline Taste transduction & 0.033 & 1 & 1 \\
\hline Cytokine-cytokine receptor interaction & 0.037 & 0.0378 & 1 \\
\hline Cell adhesion molecules (CAMs) & 0.0446 & 0.131 & 1 \\
\hline Calcium signaling pathway & 0.0506 & 0.1038 & 0.964 \\
\hline HTLV-I infection & 0.0554 & 0.2448 & 1 \\
\hline Oocyte meiosis & 0.0622 & 0.8218 & 0.53 \\
\hline Inflammatory mediator regulation of TRP channels & 0.0632 & 0.1076 & 1 \\
\hline Prion diseases & 0.0642 & 0.0066 & 1 \\
\hline TNF signaling pathway & 0.0654 & 0.1124 & 1 \\
\hline NF-kappa B signaling pathway & 0.0672 & 0.0932 & 1 \\
\hline Alzheimer's disease & 0.079 & 1 & 1 \\
\hline Amoebiasis & 0.0832 & 0.2278 & 1 \\
\hline Pyruvate metabolism & 0.0874 & 0.086 & 1 \\
\hline Amyotrophic lateral sclerosis (ALS) & 0.0892 & 1 & 1 \\
\hline Mucin type O-Glycan biosynthesis & 0.0902 & 0.0366 & 1 \\
\hline Natural killer cell mediated cytotoxicity & 0.0906 & 0.625 & 1 \\
\hline Circadian rhythm & 0.0946 & 0.096 & 1 \\
\hline Colorectal cancer & 0.0966 & 0.0354 & 1 \\
\hline NOD-like receptor signaling pathway & 0.0996 & 0.0946 & 1 \\
\hline Cocaine addiction & 0.102 & 0.608 & 0.7526 \\
\hline Apoptosis & 0.1094 & 0.4852 & 1 \\
\hline $\mathrm{T}$ cell receptor signaling pathway & 0.1172 & 0.5708 & 1 \\
\hline Measles & 0.1208 & 0.512 & 1 \\
\hline
\end{tabular}


Table 2 - Continued from previous page

\begin{tabular}{|c|c|c|c|}
\hline Pathway & $\begin{array}{l}p \text {-values } \\
\text { (semi- } \\
\text { parametric) }\end{array}$ & $\begin{array}{l}p \text {-values } \\
\text { (gamma) }\end{array}$ & $\begin{array}{l}p \text {-values } \\
\text { (fdr) }\end{array}$ \\
\hline Hippo signaling pathway & 0.121 & 0.2616 & 0.7768 \\
\hline MAPK signaling pathway & 0.1214 & 0.1802 & 0.4044 \\
\hline Salmonella infection & 0.1288 & 0.0486 & 1 \\
\hline Glycine, serine and threonine metabolism & 0.1292 & 0.0288 & 0.512 \\
\hline B cell receptor signaling pathway & 0.13 & 0.3578 & 1 \\
\hline Adipocytokine signaling pathway & 0.1312 & 0.309 & 1 \\
\hline TGF-beta signaling pathway & 0.1342 & 0.0946 & 1 \\
\hline Circadian entrainment & 0.1394 & 0.0632 & 0.7466 \\
\hline Neurotrophin signaling pathway & 0.1498 & 0.8606 & 0.5142 \\
\hline Influenza A & 0.1518 & 0.3702 & 1 \\
\hline Steroid hormone biosynthesis & 0.1558 & 1 & 1 \\
\hline Melanogenesis & 0.1582 & 0.0292 & 1 \\
\hline Amphetamine addiction & 0.1644 & 0.0952 & 0.7156 \\
\hline Malaria & 0.173 & 0.3094 & 1 \\
\hline African trypanosomiasis & 0.173 & 0.3094 & 1 \\
\hline Cysteine and methionine metabolism & 0.174 & 1 & 1 \\
\hline Inflammatory bowel disease (IBD) & 0.1746 & 1 & 1 \\
\hline Fructose and mannose metabolism & 0.1748 & 1 & 1 \\
\hline Dorso-ventral axis formation & 0.1766 & 0.0946 & 1 \\
\hline RIG-I-like receptor signaling pathway & 0.1774 & 1 & 1 \\
\hline Cytosolic DNA-sensing pathway & 0.1774 & 1 & 1 \\
\hline Sphingolipid metabolism & 0.182 & 1 & 1 \\
\hline Parkinson's disease & 0.1824 & 1 & 0.8924 \\
\hline Cell cycle & 0.19 & 0.4334 & 0.5142 \\
\hline Butanoate metabolism & 0.2126 & 0.5142 & 1 \\
\hline Vasopressin-regulated water reabsorption & 0.2214 & 0.096 & 1 \\
\hline Bacterial invasion of epithelial cells & 0.2224 & 0.5174 & 1 \\
\hline Hepatitis B & 0.23 & 0.0686 & 1 \\
\hline cGMP-PKG signaling pathway & 0.2406 & 0.0804 & 0.716 \\
\hline ErbB signaling pathway & 0.2652 & 0.4032 & 1 \\
\hline Toxoplasmosis & 0.2832 & 0.3702 & 1 \\
\hline Shigellosis & 0.2972 & 0.0486 & 1 \\
\hline Thyroid hormone synthesis & 0.299 & 0.232 & 0.784 \\
\hline Wnt signaling pathway & 0.3252 & 0.8844 & 1 \\
\hline Notch signaling pathway & 0.331 & 1 & 1 \\
\hline Basal cell carcinoma & 0.3372 & 0.5162 & 1 \\
\hline Pathways in cancer & 0.3376 & 0.3212 & 1 \\
\hline Glycosphingolipid biosynthesis - globo series & 0.3394 & 1 & 1 \\
\hline Regulation of actin cytoskeleton & 0.382 & 0.3706 & 1 \\
\hline VEGF signaling pathway & 0.4154 & 0.718 & 1 \\
\hline Leukocyte transendothelial migration & 0.4224 & 0.2996 & 1 \\
\hline Purine metabolism & 0.4236 & 1 & 0.9564 \\
\hline Bladder cancer & 0.4426 & 0.4118 & 1 \\
\hline Arachidonic acid metabolism & 0.4488 & 0.677 & 0.7718 \\
\hline Insulin signaling pathway & 0.4504 & 0.313 & 1 \\
\hline
\end{tabular}


Table 2 - Continued from previous page

\begin{tabular}{|c|c|c|c|}
\hline Pathway & $\begin{array}{l}p \text {-values } \\
\text { (semi- } \\
\text { parametric) }\end{array}$ & $\begin{array}{l}p \text {-values } \\
\text { (gamma) }\end{array}$ & $\begin{array}{l}p \text {-values } \\
\text { (fdr) }\end{array}$ \\
\hline Glutamatergic synapse & 0.4554 & 0.9532 & 0.7688 \\
\hline cAMP signaling pathway & 0.4558 & 0.0974 & 0.9612 \\
\hline AMPK signaling pathway & 0.4568 & 0.2242 & 1 \\
\hline Estrogen signaling pathway & 0.459 & 0.1794 & 1 \\
\hline Long-term potentiation & 0.4678 & 0.4244 & 0.8624 \\
\hline Fc gamma R-mediated phagocytosis & 0.4682 & 0.6288 & 0.6598 \\
\hline Oxytocin signaling pathway & 0.4716 & 0.3274 & 0.8514 \\
\hline Non-alcoholic fatty liver disease (NAFLD) & 0.479 & 1 & 1 \\
\hline Thyroid cancer & 0.4884 & 0.7138 & 1 \\
\hline Chemokine signaling pathway & 0.5262 & 0.7434 & 1 \\
\hline Adherens junction & 0.5422 & 0.3858 & 1 \\
\hline Cholinergic synapse & 0.5538 & 0.4278 & 0.5386 \\
\hline Retrograde endocannabinoid signaling & 0.5574 & 0.5982 & 1 \\
\hline Gap junction & 0.5602 & 0.7518 & 0.9534 \\
\hline Vascular smooth muscle contraction & 0.5624 & 0.6296 & 1 \\
\hline HIF-1 signaling pathway & 0.5664 & 0.3702 & 1 \\
\hline PI3K-Akt signaling pathway & 0.5668 & 0.056 & 0.7768 \\
\hline Toll-like receptor signaling pathway & 0.5708 & 0.1398 & 1 \\
\hline Inositol phosphate metabolism & 0.5916 & 1 & 1 \\
\hline Phosphatidylinositol signaling system & 0.5916 & 1 & 1 \\
\hline Chronic myeloid leukemia & 0.6112 & 0.805 & 1 \\
\hline Chagas disease (American trypanosomiasis) & 0.6184 & 0.0666 & 1 \\
\hline Lysine degradation & 0.6206 & 1 & 1 \\
\hline Focal adhesion & 0.6422 & 0.5276 & 1 \\
\hline Glycosphingolipid biosynthesis - lacto and neolacto series & 0.6526 & 1 & 1 \\
\hline ECM-receptor interaction & 0.6582 & 1 & 1 \\
\hline Renal cell carcinoma & 0.66 & 0.801 & 1 \\
\hline Prostate cancer & 0.6608 & 0.2842 & 1 \\
\hline Proteoglycans in cancer & 0.6744 & 0.5572 & 1 \\
\hline Hedgehog signaling pathway & 0.6994 & 0.7662 & 1 \\
\hline Morphine addiction & 0.7038 & 1 & 1 \\
\hline Insulin secretion & 0.708 & 0.801 & 1 \\
\hline Rap1 signaling pathway & 0.7166 & 0.763 & 0.9928 \\
\hline Thyroid hormone signaling pathway & 0.7388 & 0.6332 & 1 \\
\hline Pertussis & 0.7656 & 0.6326 & 1 \\
\hline Platelet activation & 0.7762 & 0.4428 & 1 \\
\hline Jak-STAT signaling pathway & 0.7918 & 1 & 1 \\
\hline Signaling pathways regulating pluripotency of stem cells & 0.7926 & 0.6508 & 1 \\
\hline Epstein-Barr virus infection & 0.7936 & 1 & 1 \\
\hline Type II diabetes mellitus & 0.794 & 0.6974 & 1 \\
\hline GnRH signaling pathway & 0.8004 & 0.2552 & 1 \\
\hline mTOR signaling pathway & 0.8034 & 0.5968 & 1 \\
\hline Adrenergic signaling in cardiomyocytes & 0.8068 & 0.0134 & 0.9956 \\
\hline GABAergic synapse & 0.8304 & 0.7428 & 1 \\
\hline beta-Alanine metabolism & 0.8356 & 0.6766 & 0.5264 \\
\hline
\end{tabular}


Table 2 - Continued from previous page

\begin{tabular}{|c|c|c|c|}
\hline Pathway & $\begin{array}{l}p \text {-values } \\
\text { (semi- } \\
\text { parametric) }\end{array}$ & $\begin{array}{l}p \text {-values } \\
\text { (gamma) }\end{array}$ & $\begin{array}{l}p \text {-values } \\
\text { (fdr) }\end{array}$ \\
\hline Drug metabolism - other enzymes & 0.8356 & 0.6766 & 0.5264 \\
\hline Fc epsilon RI signaling pathway & 0.8394 & 0.5604 & 1 \\
\hline Acute myeloid leukemia & 0.8416 & 0.5968 & 1 \\
\hline Non-small cell lung cancer & 0.8416 & 0.5968 & 1 \\
\hline Pancreatic cancer & 0.8416 & 0.5968 & 1 \\
\hline Endometrial cancer & 0.8416 & 0.5968 & 1 \\
\hline Prolactin signaling pathway & 0.8442 & 0.5606 & 1 \\
\hline MicroRNAs in cancer & 0.8496 & 0.1518 & 0.3328 \\
\hline Pantothenate and CoA biosynthesis & 0.864 & 0.7748 & 0.5264 \\
\hline Tight junction & 0.8834 & 0.17 & 1 \\
\hline Long-term depression & 0.8862 & 0.4218 & 0.5324 \\
\hline Dopaminergic synapse & 0.8868 & 0.015 & 0.925 \\
\hline Glioma & 0.9018 & 0.5968 & 1 \\
\hline Progesterone-mediated oocyte maturation & 0.9018 & 0.7476 & 1 \\
\hline FoxO signaling pathway & 0.9022 & 0.7088 & 1 \\
\hline Serotonergic synapse & 0.9106 & 0.9062 & 1 \\
\hline Hepatitis C & 0.9122 & 0.1406 & 1 \\
\hline Pyrimidine metabolism & 0.9238 & 0.9284 & 0.5336 \\
\hline Dilated cardiomyopathy & 0.9456 & 1 & 1 \\
\hline Melanoma & 0.957 & 0.7938 & 1 \\
\hline Alcoholism & 0.9636 & 0.9998 & 0.0822 \\
\hline Olfactory transduction & 0.9852 & 0.9458 & 0.9826 \\
\hline Ras signaling pathway & 0.9864 & 0.8614 & 1 \\
\hline Pathogenic Escherichia coli infection & 1 & 0.092 & 1 \\
\hline N-Glycan biosynthesis & 1 & 0.1164 & 0.2634 \\
\hline Pentose and glucuronate interconversions & 1 & 0.3054 & 1 \\
\hline Epithelial cell signaling in Helicobacter pylori infection & 1 & 0.4482 & 1 \\
\hline Alanine, aspartate and glutamate metabolism & 1 & 0.5142 & 1 \\
\hline Aminoacyl-tRNA biosynthesis & 1 & 0.7708 & 0.8938 \\
\hline alpha-Linolenic acid metabolism & 1 & 1 & 1 \\
\hline Graft-versus-host disease & 1 & 1 & 1 \\
\hline Histidine metabolism & 1 & 1 & 1 \\
\hline Huntington's disease & 1 & 1 & 1 \\
\hline Hypertrophic cardiomyopathy (HCM) & 1 & 1 & 1 \\
\hline Intestinal immune network for IgA production & 1 & 1 & 1 \\
\hline Legionellosis & 1 & 1 & 1 \\
\hline Linoleic acid metabolism & 1 & 1 & 1 \\
\hline Lipoic acid metabolism & 1 & 1 & 1 \\
\hline Lysine biosynthesis & 1 & 1 & 1 \\
\hline Amino sugar and nucleotide sugar metabolism & 1 & 1 & 1 \\
\hline Maturity onset diabetes of the young & 1 & 1 & 1 \\
\hline Metabolism of xenobiotics by cytochrome P450 & 1 & 1 & 1 \\
\hline Mineral absorption & 1 & 1 & 1 \\
\hline Neuroactive ligand-receptor interaction & 1 & 1 & 1 \\
\hline Nicotinate and nicotinamide metabolism & 1 & 1 & 0.7818 \\
\hline
\end{tabular}


Table 2 - Continued from previous page

\begin{tabular}{|c|c|c|c|}
\hline Pathway & $\begin{array}{l}p \text {-values } \\
\text { (semi- } \\
\text { parametric) }\end{array}$ & $\begin{array}{l}p \text {-values } \\
\text { (gamma) }\end{array}$ & $\begin{array}{l}p \text {-values } \\
\text { (fdr) }\end{array}$ \\
\hline Nitrogen metabolism & 1 & 1 & 1 \\
\hline One carbon pool by folate & 1 & 1 & 1 \\
\hline Ovarian steroidogenesis & 1 & 1 & 1 \\
\hline Oxidative phosphorylation & 1 & 1 & 1 \\
\hline p53 signaling pathway & 1 & 1 & 1 \\
\hline Pancreatic secretion & 1 & 1 & 1 \\
\hline Phenylalanine metabolism & 1 & 1 & 1 \\
\hline Phenylalanine, tyrosine and tryptophan biosynthesis & 1 & 1 & 1 \\
\hline Phototransduction & 1 & 1 & 1 \\
\hline Porphyrin and chlorophyll metabolism & 1 & 1 & 1 \\
\hline Primary bile acid biosynthesis & 1 & 1 & 1 \\
\hline Propanoate metabolism & 1 & 1 & 1 \\
\hline Proximal tubule bicarbonate reclamation & 1 & 1 & 1 \\
\hline Retinol metabolism & 1 & 1 & 1 \\
\hline Rheumatoid arthritis & 1 & 1 & 1 \\
\hline Riboflavin metabolism & 1 & 1 & 1 \\
\hline Salivary secretion & 1 & 1 & 1 \\
\hline Selenocompound metabolism & 1 & 1 & 1 \\
\hline Small cell lung cancer & 1 & 1 & 1 \\
\hline Staphylococcus aureus infection & 1 & 1 & 1 \\
\hline Starch and sucrose metabolism & 1 & 1 & 1 \\
\hline Steroid biosynthesis & 1 & 1 & 1 \\
\hline Sulfur metabolism & 1 & 1 & 1 \\
\hline Synaptic vesicle cycle & 1 & 1 & 1 \\
\hline Arginine and proline metabolism & 1 & 1 & 1 \\
\hline Synthesis and degradation of ketone bodies & 1 & 1 & 1 \\
\hline Systemic lupus erythematosus & 1 & 1 & 1 \\
\hline Taurine and hypotaurine metabolism & 1 & 1 & 1 \\
\hline Terpenoid backbone biosynthesis & 1 & 1 & 1 \\
\hline Thiamine metabolism & 1 & 1 & 1 \\
\hline $\begin{array}{l}\text { Arrhythmogenic right ventricular cardiomyopathy } \\
\text { (ARVC) }\end{array}$ & 1 & 1 & 1 \\
\hline Transcriptional misregulation in cancer & 1 & 1 & 1 \\
\hline Tryptophan metabolism & 1 & 1 & 1 \\
\hline Type I diabetes mellitus & 1 & 1 & 1 \\
\hline Asthma & 1 & 1 & 1 \\
\hline Tyrosine metabolism & 1 & 1 & 1 \\
\hline Ubiquinone and other terpenoid-quinone biosynthesis & 1 & 1 & 1 \\
\hline Valine, leucine and isoleucine biosynthesis & 1 & 1 & 1 \\
\hline Valine, leucine and isoleucine degradation & 1 & 1 & 1 \\
\hline Vibrio cholerae infection & 1 & 1 & 0.7708 \\
\hline Viral carcinogenesis & 1 & 1 & 1 \\
\hline Viral myocarditis & 1 & 1 & 1 \\
\hline Autoimmune thyroid disease & 1 & 1 & 1 \\
\hline Vitamin B6 metabolism & 1 & 1 & 1 \\
\hline
\end{tabular}


Table 2 - Continued from previous page

\begin{tabular}{|c|c|c|c|}
\hline Pathway & $\begin{array}{l}p \text {-values } \\
\text { (semi- } \\
\text { parametric) }\end{array}$ & $\begin{array}{l}p \text {-values } \\
\text { (gamma) }\end{array}$ & $\begin{array}{l}p \text {-values } \\
\text { (fdr) }\end{array}$ \\
\hline Vitamin digestion and absorption & 1 & 1 & 1 \\
\hline Bile secretion & 1 & 1 & 1 \\
\hline Biotin metabolism & 1 & 1 & 1 \\
\hline Butirosin and neomycin biosynthesis & 1 & 1 & 1 \\
\hline Caffeine metabolism & 1 & 1 & 1 \\
\hline Carbohydrate digestion and absorption & 1 & 1 & 1 \\
\hline Cardiac muscle contraction & 1 & 1 & 1 \\
\hline Chemical carcinogenesis & 1 & 1 & 1 \\
\hline Citrate cycle (TCA cycle) & 1 & 1 & 1 \\
\hline Complement and coagulation cascades & 1 & 1 & 1 \\
\hline Cyanoamino acid metabolism & 1 & 1 & 1 \\
\hline D-Glutamine and D-glutamate metabolism & 1 & 1 & 1 \\
\hline Drug metabolism - cytochrome P450 & 1 & 1 & 1 \\
\hline $\begin{array}{l}\text { Endocrine and other factor-regulated calcium reabsorp- } \\
\text { tion }\end{array}$ & 1 & 1 & 1 \\
\hline Ether lipid metabolism & 1 & 1 & 1 \\
\hline Fat digestion and absorption & 1 & 1 & 1 \\
\hline Fatty acid biosynthesis & 1 & 1 & 1 \\
\hline Fatty acid degradation & 1 & 1 & 1 \\
\hline Fatty acid elongation & 1 & 1 & 1 \\
\hline Folate biosynthesis & 1 & 1 & 1 \\
\hline Aldosterone-regulated sodium reabsorption & 1 & 1 & 1 \\
\hline Galactose metabolism & 1 & 1 & 1 \\
\hline Gastric acid secretion & 1 & 1 & 0.8974 \\
\hline Glutathione metabolism & 1 & 1 & 1 \\
\hline Glycerolipid metabolism & 1 & 1 & 1 \\
\hline Allograft rejection & 1 & 1 & 1 \\
\hline Glycerophospholipid metabolism & 1 & 1 & 1 \\
\hline Glycolysis / Gluconeogenesis & 1 & 1 & 1 \\
\hline $\begin{array}{l}\text { Glycosaminoglycan biosynthesis - chondroitin sulfate / } \\
\text { dermatan sulfate }\end{array}$ & 1 & 1 & 1 \\
\hline Glycosaminoglycan degradation & 1 & 1 & 1 \\
\hline Glycosphingolipid biosynthesis - ganglio series & 1 & 1 & 1 \\
\hline Glycosylphosphatidylinositol(GPI)-anchor biosynthesis & 1 & 1 & 1 \\
\hline Glyoxylate and dicarboxylate metabolism & 1 & 1 & 1 \\
\hline
\end{tabular}

\section{Supplement C: Identifiability of the mixture model.}

First consider the model without covariates. At the global level, the twocomponent mixture model under the symmetric assumption has marginal density $f(|z|)=\left(1-\pi_{1}\right) f_{0}(|z|)+\pi_{1} f_{1}(|z|)$, where $f_{0}$ is a folded $N\left(0, \sigma_{0}^{2}\right), f_{1}$ is a nonparametric non-null distribution with known location $\mu \geq 0.68$, and 
$\pi_{1}$ takes the form of the logistic function $\frac{\exp (\gamma)}{1+\exp (\gamma)}$. This model is identifiable upon making the center null assumption for $f_{0}$ and "zero assumption" of Efron (2007). At the local level, the non-null density is approximated by cubic B-spline density $g_{k}(|z|)$ and mixing weight $c_{k}$ such that $f_{1}(|z|)=$ $\sum_{k=1}^{K} c_{k} g_{k}(|z|)$. The mixing weight $c_{k}$ is a multinomial function $\frac{\exp \left(\boldsymbol{\alpha}_{k}\right)}{\sum_{l=1}^{K} \exp \left(\boldsymbol{\alpha}_{l}\right)}$ where $\sum_{k=1}^{K} c_{k}=1$. According to Inkila (1988) and De Boor et al. (1978), a sequence of cubic B-splines generated over a strictly increasing sequence of knots is uniquely defined. The overall model is thus identifiable in the case of no covariates.

From here, identifiability of our covariate-modulated model follows in an analogous manner to Theorem 1 of Huang and Yao (2012). In particular, the covariates $\boldsymbol{x}$ enter our model through $\boldsymbol{x}^{T} \boldsymbol{\gamma}$ and $\boldsymbol{x}^{T} \boldsymbol{\alpha}$ components in the mixing weights $\pi_{1}$ and $c_{k}$ respectively. The model conditioning on the covariates $\boldsymbol{x}$ is identifiable. Assuming the domain of the covariates $\boldsymbol{x}$ has no isolated points, identifiability of our proposed mixture model, then follows through in an identical manner to the proof of Theorem 1 of Huang and Yao (2012).

\section{Supplement D: Convergence Diagnosis Plots.}



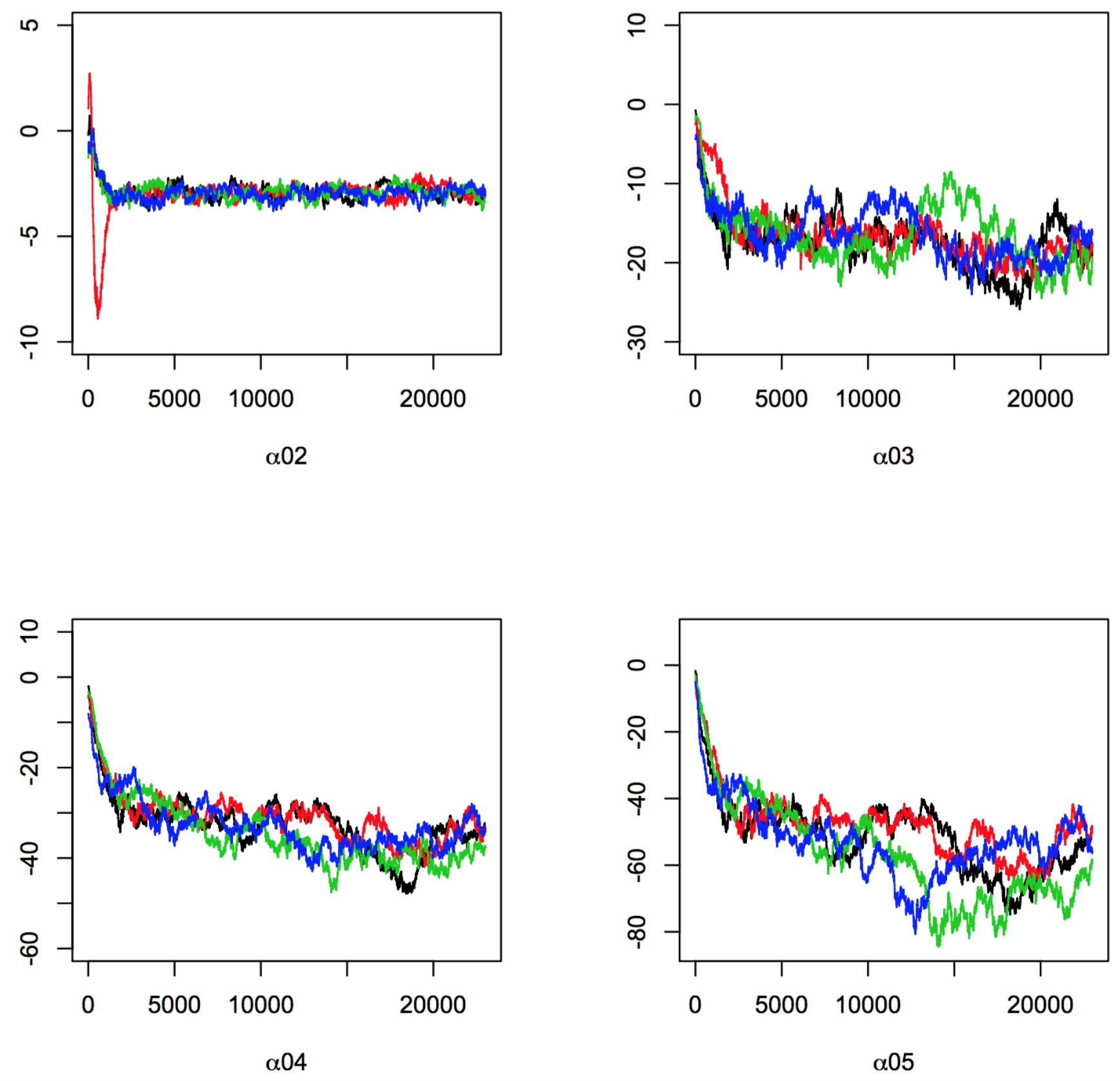

Fig 1: Running mean plot of $\boldsymbol{\alpha}_{\mathbf{0}}$, 23000 iterations, 4 chains. (note: $\boldsymbol{\alpha}_{01}=$ $0)$. 

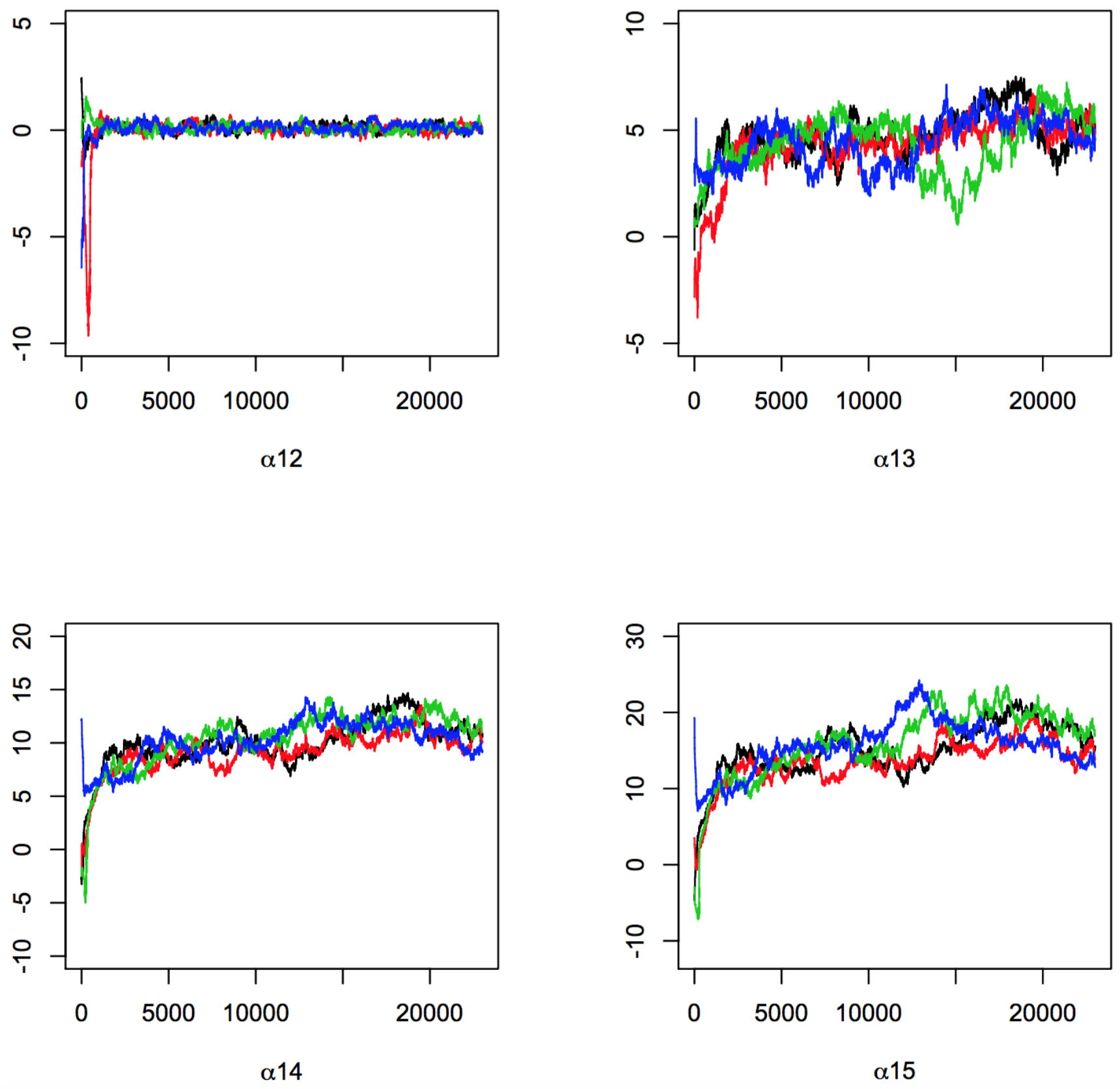

Fig 2: Running mean plot of $\boldsymbol{\alpha}_{\mathbf{1}}$, 23000 iterations, 4 chains. (note: $\boldsymbol{\alpha}_{11}=$ $0)$. 

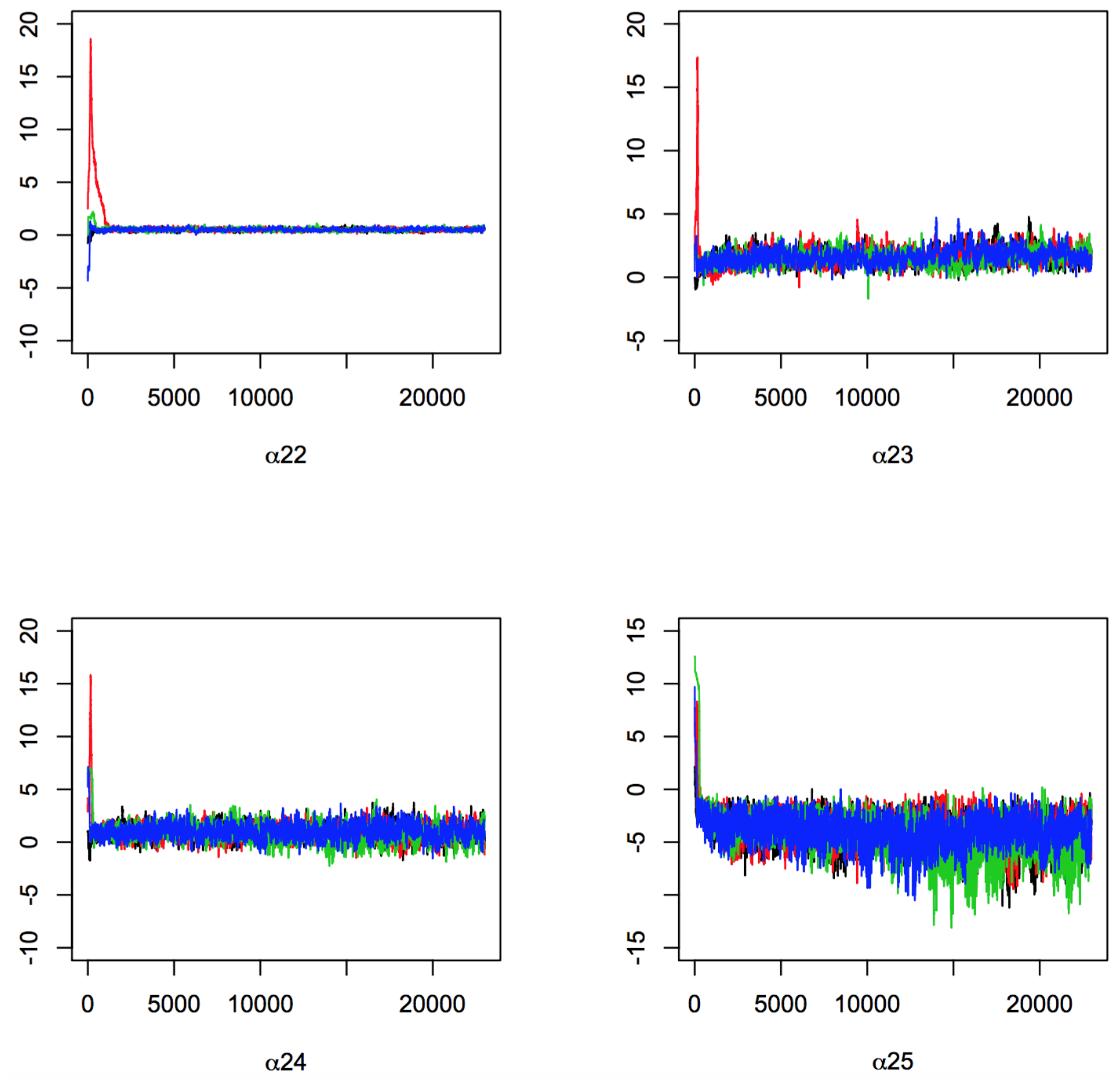

Fig 3: Running mean plot of $\boldsymbol{\alpha}_{\mathbf{2}}$, 23000 iterations, 4 chains. (note: $\boldsymbol{\alpha}_{21}=$ $0)$. 

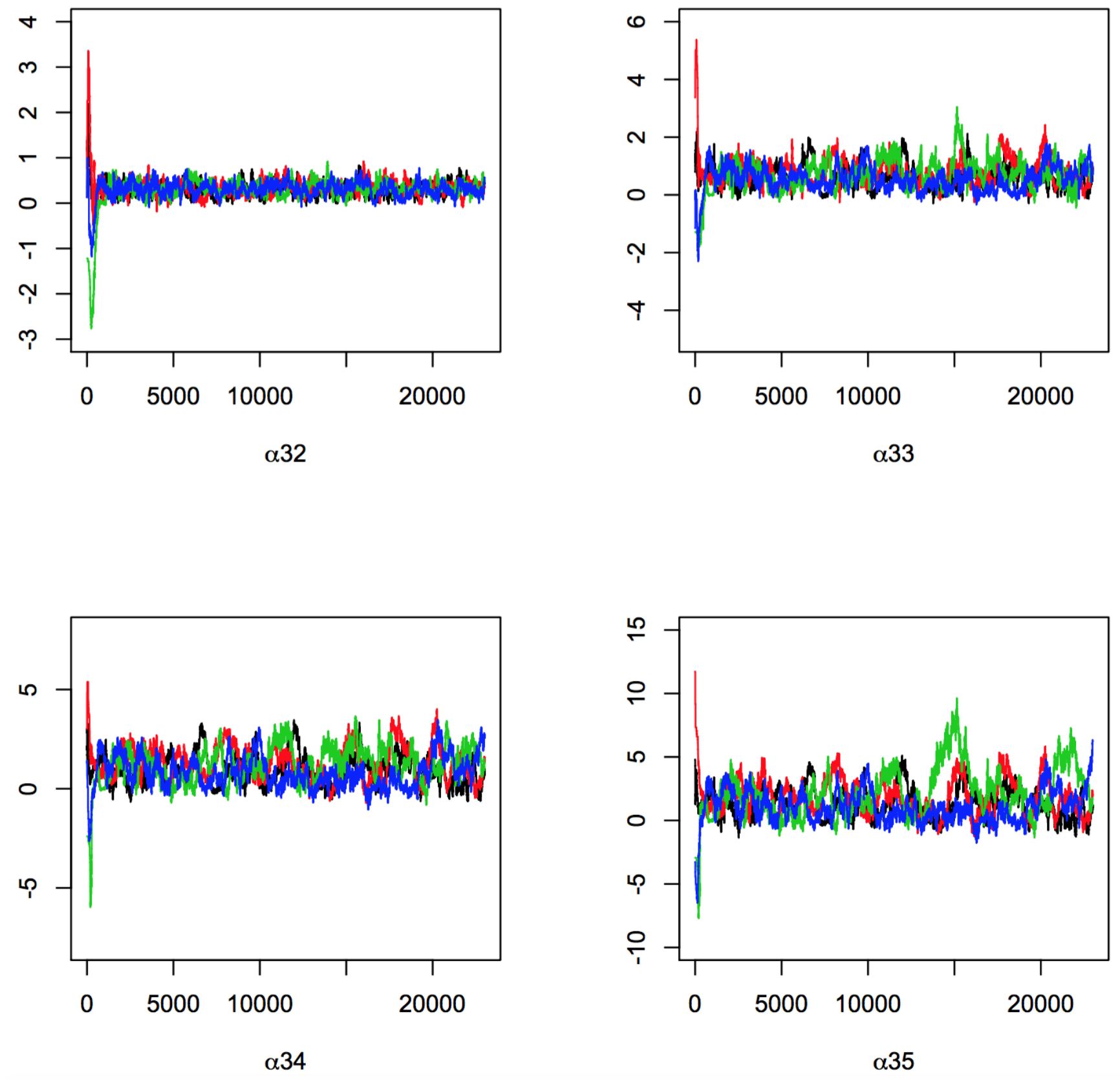

Fig 4: Running mean plot of $\boldsymbol{\alpha}_{\mathbf{3}}$., 23000 iterations, 4 chains. (note: $\boldsymbol{\alpha}_{31}=$ $0)$. 

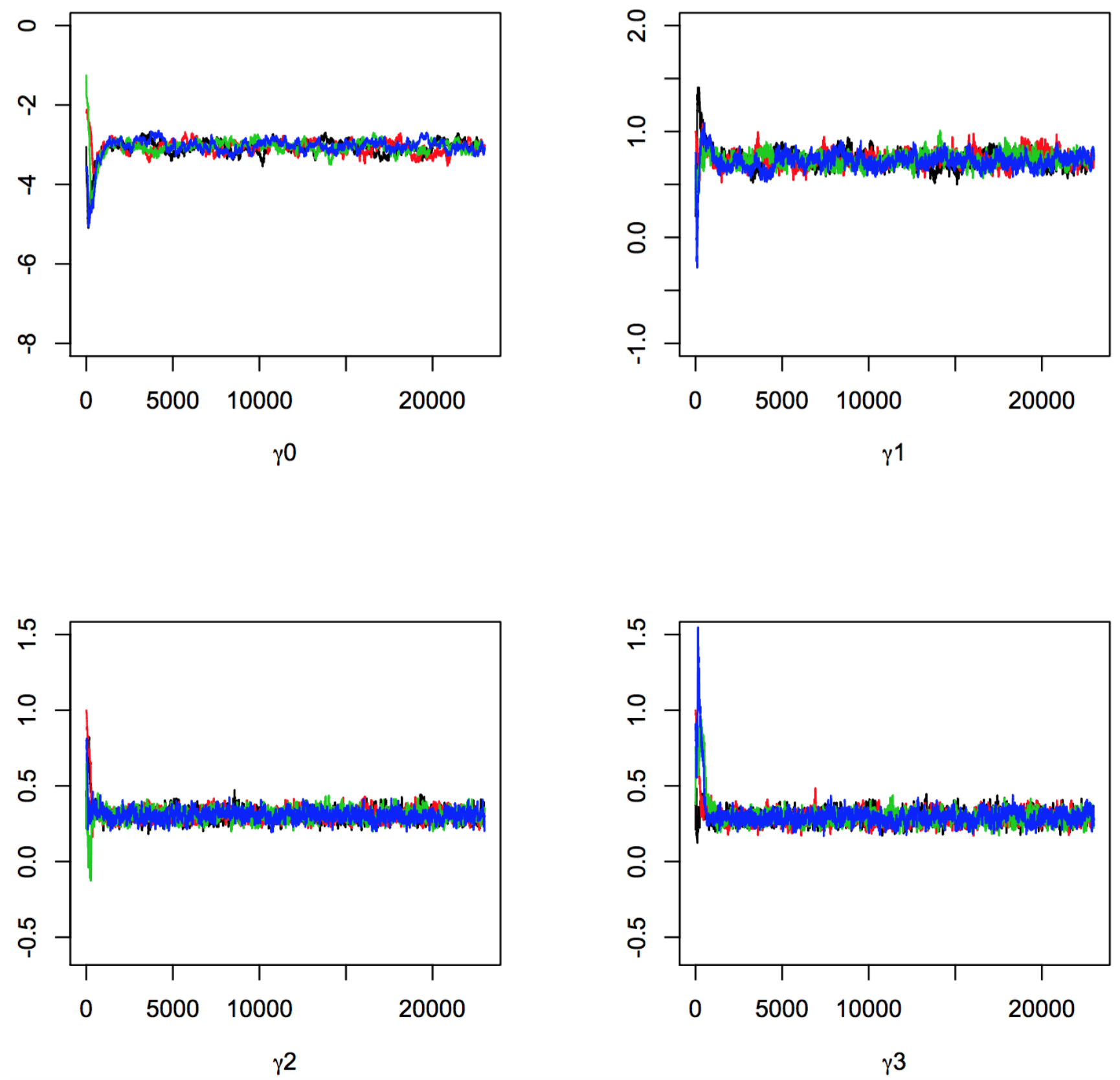

Fig 5: Running mean plot of $\boldsymbol{\gamma}, 23000$ iterations, 4 chains. 


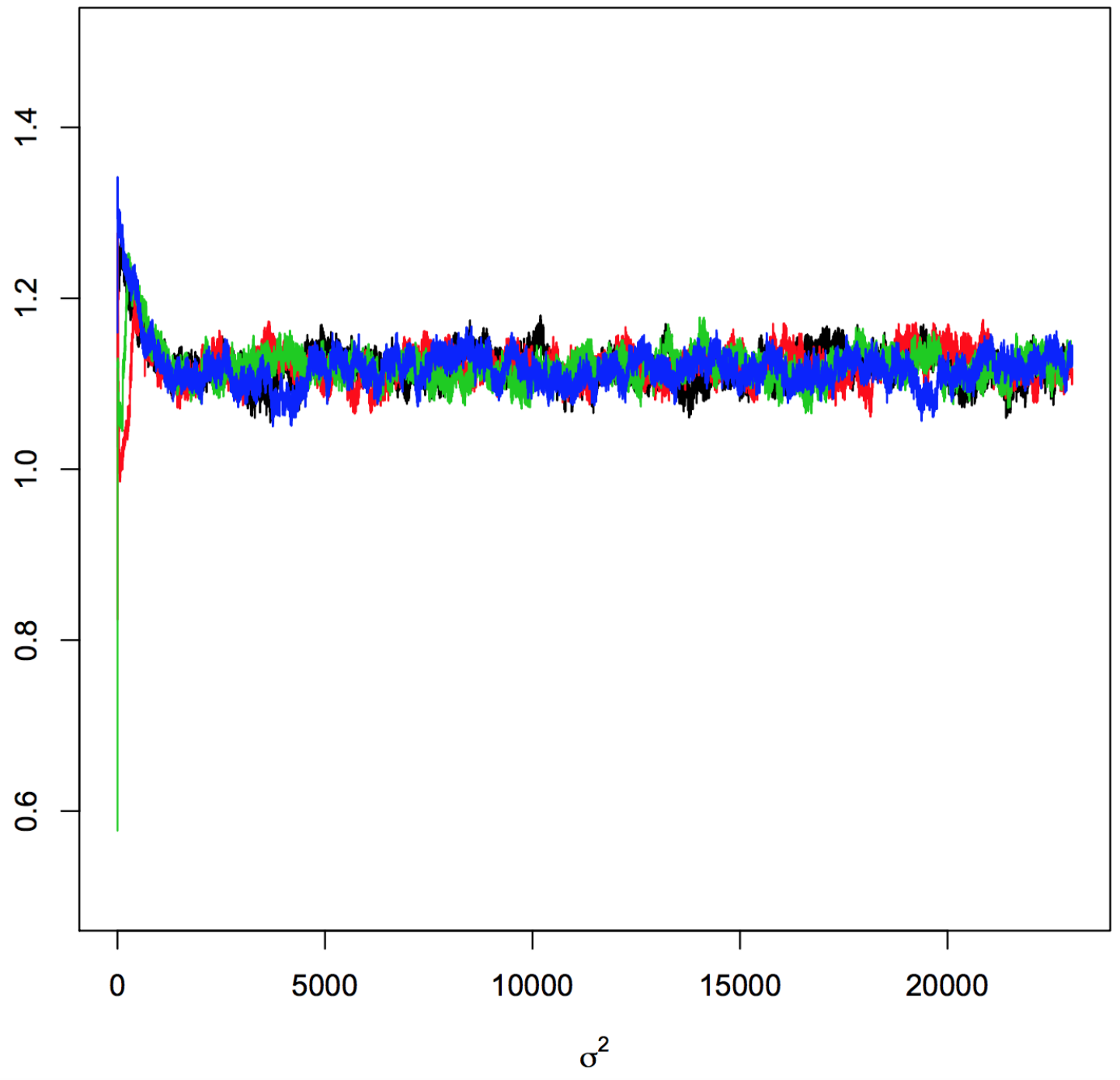

Fig 6: Running mean plot of $\sigma_{0}^{2}, 23000$ iterations, 4 chains. 


\section{References.}

De Boor, C., De Boor, C., Mathématicien, E.-U., De Boor, C. and De Boor, C. (1978). A practical guide to splines 27. Springer-Verlag New York.

Efron, B. (2007). Size, power and false discovery rates. The Annals of Statistics 13511377.

Givens, G. H. and Hoeting, J. A. (2005). Computational statistics 483. Wiley Interscience Press.

HuAnG, M. and YAO, W. (2012). Mixture of regression models with varying mixing proportions: a semiparametric approach. Journal of the American Statistical Association $107711-724$.

INKILA, K. (1988). Bicubic B-spline approximation by least squares. In XVIth ISPRS Congress, Technical Commission III: Mathematical Analysis of Data XXVII Part B3 $281-287$.

R Core Team (2016). R: A Language and Environment for Statistical Computing R Foundation for Statistical Computing, Vienna, Austria.

RONG W. ZABLOCKI

Computational Science Research Center

San Diego State University

5500 Campanile Drive

SAN Diego, CA 92182

USA;

Institute of Mathematical Sciences

Claremont Graduate University

150 E. 10TH ST.

Claremont, CA 91711

USA

ANDREW J. SCHORK

Cognitive Sciences Graduate Program

University of California at San Diego

9500 Gilman Drive

LA Jolla, CA 92093

USA

YunPENG WANG

Institute of Clinical Medicine

UNIVERSITY OF OSLO

OSLO, 0424

NORWAY
Richard A. Levine

Department of Mathematics and Statistics San Diego State University

5500 Campanile Drive

San Diego, CA 92182

USA

\author{
Shujing Xu \\ Department of Psychiatry \\ University of California at San Diego \\ 9500 Gilman Drive \\ LA Jolla, CA 92093 \\ USA \\ Chun C. Fan \\ Cognitive Sciences Graduate Program \\ University of California at San Diego \\ 9500 Gilman Drive \\ LA Jolla, CA 92093 \\ USA
}

Wesley K. Thompson

InStitute of Biological Psychiatry

Mental Health Centre Sct. Hans

Mental Health Services Copenhagen

DK-4000

DENMARK

DEPARTMENT OF PSychiatry

University of California at San Diego

9500 Gilman Drive

LA Jolla, CA 92093

USA E-MAIL: wes.stat@gmail.com 